\title{
Effects of organic matter degradation in cohesive sediment: linking sediment rheology to spatio-temporal patterns of organic matter degradability
}

\author{
F. Zander ${ }^{1}$ (D) A. Shakeel ${ }^{2,3} \cdot$ A. Kirichek ${ }^{2} \cdot$ C. Chassagne $^{2} \cdot$ J. Gebert $^{1}$
}

Received: 12 October 2021 / Accepted: 30 January 2022 / Published online: 12 February 2022

(c) The Author(s) 2022

\begin{abstract}
Purpose Sediment organic matter (SOM) influences settling and thus the rheological behavior of suspended particles by enhancing flocculation or reducing surface charges by forming organo-mineral complexes that facilitate particle-particle interactions in consolidating sediments. It was, therefore, assumed that the microbial degradation of SOM and its spatio-temporal variability would affect sediment rheological properties and enhance port maintenance dredging and navigability of ports and waterways. Methods To investigate this effect, samples were taken at six locations along a transect of 30 river kilometers through the Port of Hamburg, Germany, during nine sampling campaigns within two years. The collected samples were divided into different layers based on the differences in visual consistency and strength. For analysis of SOM degradability, the samples were incubated in the laboratory for 250 days in glass bottles under aerobic and anaerobic conditions following the evolution of gas composition $(\mathrm{CH} 4, \mathrm{CO} 2)$ and pressure in the bottle headspace over time. Yield stress was analyzed before and after the dissolved organic matter (DOM) decay using a rheometer with Couette geometry. Standard properties of solids and pore water were also analyzed.

Results Shear strength decreased upon SOM decay under both anaerobic and aerobic conditions. Under anaerobic conditions, organic matter decay reduced static and fluidic yield stresses to an average of $74 \%$ and $79 \%$ of the fresh sample values. Consolidated layers at lower depths showed the highest absolute decrease in fluidic yield stress of up to - 110 Pa due to a larger absolute amount of degradable organic matter in these layers in connection to higher bulk density. Pronounced spatial trends with higher changes in yield stress at upstream locations and lower yield stress changes at downstream locations coincided with a decreasing gradient of SOM degradability from upstream to downstream. Seasonal trends indicated that the investigation area is impacted by temporally changing factors.

Conclusion The availability of easily degradable organic matter significantly affects sediment strength, especially under the anaerobic conditions, even when the mass loss of organic matter mass loss is small. Seasonal variability in yield stress changes upon SOM decay indicate that the site-specific responses were modulated by overarching seasonal effects impacting the entire investigation area. It was assumed that during an anaerobic decay, the formation of gas bubbles added an additional physical component to the effect of biological SOM decay.
\end{abstract}

Keywords River sediment · Organic matter degradation $\cdot$ Gas production Respiration · Yield stress

\section{Introduction}

Sediment organic matter (SOM) originates from both natural (e.g., eroded terrestrial topsoils, plant litter, planktonic, and benthic organisms) and anthropogenic (e.g., surface runoff,

Responsible editor: Jasmina Obhodas

F. Zander

f.zander@tudelft.nl

Extended author information available on the last page of the article sewage waste) sources. The presence of organic matter can expedite suspended particle settling by flocculation through bridging between the mineral particles, creating larger diameter particles or by surface charge neutralization, reducing the repulsive forces between the charged particles (Lagaly and Dékány 2013). As a result, the sediment strength increases with increasing amount of organic matter at the same bulk density (Shakeel et al. 2019).

Phytoplankton can play a significant role in the settling of fine-grained sediment by facilitating the bridging effect 
(Deng et al. 2019). Correspondingly, fine-grained sediment is expected to behave differently in shallow areas, upstream within the river continuum (Vannote et al. 1980) of high net primary production compared to light-deficient areas in deeper and/or more turbulent waters. The existence of organic matter in sediments is known to significantly influence the rheological and cohesive properties of sediments (Wurpts and Torn 2005; Shakeel et al. 2019).

As part of the natural carbon cycle, the labile part of SOM is also subject to microbial degradation. Under aerobic conditions, SOM degradation results in the production of carbon dioxide $\left(\mathrm{CO}_{2}\right)$, while under anaerobic conditions, frequently prevailing in fine-grained sediments, methane $\left(\mathrm{CH}_{4}\right)$ is also produced. Particularly, $\mathrm{CH}_{4}$ can remain entrapped in the sediment and affect its rheological properties and density due to the poor solubility of methane in water. The non-degraded part of SOM forms complexes with the mineral phase, leading to its stabilization and physical shielding and transfer of SOM into the heavy density fraction (Baldock and Skjemstad 2000; Six and Paustian 2014; Gao et al. 2019; Zander et al. 2020). Given the role of organic matter for the sediment's rheological properties, it can be expected that, next to the absolute SOM content, its degradability (inversely) influences these properties. In the investigated transect through the Port of Hamburg in the tidal Elbe river, SOM decay has been shown to follow trends in space, in time, and with depth (Zander et al. 2020).

It is therefore hypothesized that:

1. Sediment yield stresses are influenced by sediment organic matter decay with seasonal and spatial trends.

2. The rheology is dependent on the sediment density and the amount of decayed sediment organic matter.

3. Anaerobically decayed samples display greater changes in rheological properties than aerobically decayed samples due to bubble formation.

\section{Materials and methods}

In this study, natural sediment samples were collected from different locations along a transect of around 30 river kilometers $(\mathrm{P} 1=$ river $\mathrm{km} \mathrm{616}, \mathrm{P} 9=$ river $\mathrm{km} \mathrm{646,} \mathrm{Fig.} \mathrm{S1,}$ supporting information) through the Port of Hamburg (site description found in Zander et al. 2020) using a $1 \mathrm{~m}$ core sampler ("Frahmlot") in the years 2019-2020. The collected samples were divided into different stratigraphic layers (fluid mud, pre-consolidated, and consolidated sediment) based on the differences in their visual consistency and strength for further analysis. The layers did not show constant thickness, but always had the same stratigraphic order as the FM layer that was not always present.

\subsection{Overview of material properties}

Methods for standard properties of solids and pore water are given in Zander et al. (2020). Some basic properties of the sediments investigated in this study are summarized in Table S1 (supporting information). Minimum and maximum values of selected parameters were total nitrogen (TN, 0.3-0.9\%, downstream location P9, and upstream location P1), total organic carbon (TOC, 2.4-6.3\%, P9 and P1), clay (31-43\%, P5 and P8), sand (5-27\%, P1 and P5), and extracellular polymeric substance (EPS, 2-32 $\mathrm{g} \mathrm{kg}_{\mathrm{DM}}{ }^{-1}$, $\mathrm{P} 9$ and $\mathrm{P} 1$ ), highlighting the pronounced spatial gradient found along the transect. Negative redox potentials indicate anaerobic conditions at all the investigated sites.

\subsection{Impact of SOM decay on rheological properties}

Degradation of sediment organic matter was performed by using the methodology reported in Zander et al. (2020). Sediment samples were incubated for 250 days in glass bottles under aerobic and anaerobic conditions, measuring the $\mathrm{CO}_{2}$ and $\mathrm{CH}_{4}$ concentrations with gas chromatographic analyses and the pressure increase in the bottle headspace. From these, the amounts of released $\mathrm{CO}_{2}-\mathrm{C}$ and $\mathrm{CH}_{4}-\mathrm{C}$ were calculated and normalized to total organic carbon (TOC). Before and after the 250 days of incubation, rheological analyses were performed.

\subsection{Rheological characterization}

Static yield stress was obtained from the first decline in viscosity as a function of shear stress, which is linked to the breakage of the inter-connected network of flocs. On the other hand, the second decline in viscosity (i.e., fluidic yield stress) was associated to the breakage of reorganized flocs into smaller flocs or individual particles upon further shearing (Shakeel et al. 2022). The rheological analysis of both fresh and degraded mud samples was performed using a HAAKE MARS I rheometer (Thermo Scientific, Germany) with concentric cylinder (Couette) geometry (gap width $=1 \mathrm{~mm}$ ). The mud samples were gently homogenized before each rheological experiment, and a waiting time of 3-5 min was adopted before the test to minimize the disturbances created by the cylinder. The rheological experiments were performed at $20^{\circ} \mathrm{C}$, maintained by a Peltier controller system. In order to check the repeatability, all the experiments were performed in duplicate, and the repeatability error was less than 2\%. More details can be found in Shakeel et al. (2019).

Density and water contents were measured directly before analyzing the yield stress. The samples' moisture did, in principle, not change within the air tight incubation 
containers and were checked by weighing the containers. If water was lost, for example, due to re-flushing of samples with air during aerobic incubation, demineralized water was added to compensate for this loss before yield stresses were measured. If the water content changes (fresh sample compared to degraded sample after the rheological analyses) still was higher than 5\%, the sample was discarded since density strongly influence the yield stress.

All investigated samples showed a two-step yielding behavior, with the two yield points defined as static $\tau_{s}$ and fluidic yield stress $\tau_{f}$ (Shakeel et al. 2020a). The change in yield stress is the difference between the initial yield stress (fresh sample, $\tau_{s, f f r e s h}$ ) and the yield stress of degraded samples $\left(\tau_{s, f \_d e g}\right)$ with $\tau_{s, f}=$ static and fluidic yield stress. In this paper, this change is expressed as follows:

$\Delta \tau_{s, f}=\tau_{s, f_{-} d e g}-\tau_{s, f_{\text {fresh }}}$

The percentage of the degraded sample's yield stress related to the fresh sample's yield stress is given by:

$\frac{\tau_{s, f_{-} d e g}}{\tau_{s, f_{\text {fresh }}}} \times 100(\%)$

and the percentage of yield stress change in relation to the original value (fresh sample) by:

$\frac{\left|\Delta \tau_{s, f}\right|}{\tau_{s, f f r e s h}} \times 100(\%)$

\section{Results}

\subsection{Effect of organic matter decay on sediment yield stress}

Cumulative degradation of sediment organic matter followed a multiphase exponential decay function (Zander et al. 2020). After 250 days of laboratory incubation, degradation rates for most samples were extremely low, meaning that the cumulative increase of released carbon reached a plateau. The loss of organic carbon during this exhaustive laboratory incubation under anaerobic conditions ranged between 30 and $215 \mathrm{mg} \mathrm{C}_{\mathrm{TOC}^{-}}{ }^{-1}$, i.e., between 3 and $21.5 \%$ of the organic carbon was available for microbial degradation (minimum and maximum, Fig. 1, top left). Under aerobic conditions, the maximum release of organic carbon ranged between 91 and $344 \mathrm{mg} \mathrm{C} \mathrm{g}_{\text {TOC }}{ }^{-1}$, i.e., 9.1 and 34.4\% (Fig. 1, top right). In situ, anaerobic conditions are dominant, indicated by negative redox potentials (see Table S1, supporting information).

For the vast majority of samples, organic matter degradation under anaerobic conditions led to a decline in strength, and therefore in static and fluidic yield stresses as characteristic values for a two-step yielding behavior (Shakeel et al. 2020a). On average, the yield stresses of anaerobically decayed SOM decreased to $74 \%$ of the original value for static yield stress (SYS) and to $79 \%$ in case of the fluidic yield stress (FYS, both Fig. 1, bottom). For aerobically decayed samples, a less strong effect was observed, i.e., on average a decrease to $93 \%$ of the original value for SYS and to $98 \%$ for FYS (note: $100 \%$ means that the decayed sample had the same YS value than the initial sample). For some samples, organic matter decay led to an increase in strength (values $>100 \%$ in Fig. 1, bottom). The overall variability of yield stress values was much larger for aerobic than for anaerobic SOM decay.

\subsection{Spatial and seasonal variation of yield stresses}

Independent of sampling location or sampling depth (layer), it was found that the higher the strength of the fresh sample, the larger the decrease in strength upon degradation of organic matter, shown for the fluidic yield stress in Fig. 2. As carbon content and degradability are subject to both a seasonal variation and a spatial trend (Zander et al. 2020), it was hypothesized that these patterns would also be reflected in the effect of organic matter decay on the rheological properties.

If the dataset is divided by date of sampling (Fig. 2, left), with each sampling date including all sampled locations and layers, it is seen that the ratio between the initial yield stresses and the change of yield stresses are reversed for each date with high Pearson's coefficients $(P \geq 0.85)$ except for the campaign in June 2020 (20-jun, $P:-0.59$ ). This suggests that the sediment properties at all locations along the transect follow a similar principal temporal trend. If the dataset is divided by location of sampling (Fig. 2, right), including all sampling dates for each location, a similar inverse relationship between initial yield stresses and the change of yield stresses is seen, with even higher Pearson's coefficients $(P \geq 0.9)$, except for the location P8 $(P:-0.75)$. For most of the samples, the slopes of change in yield stress over initial yield stress were more negative (steeper) in 2019 than in 2020 (Fig. 2, left) and more negative at upstream compared to downstream locations ( $\mathrm{P} 1$ and $\mathrm{P} 2$, Fig. 2, right). This suggests that the sediment properties at each location differ distinctly from one another and also maintain these differences over time. A similar pattern could be observed for the static yield stress (not shown here). Trends were less pronounced for aerobically decayed samples.

\subsection{Depth-differentiation of yield stresses}

If the data set is differentiated per depth, clear differences for the change in yield stress can be observed between the 

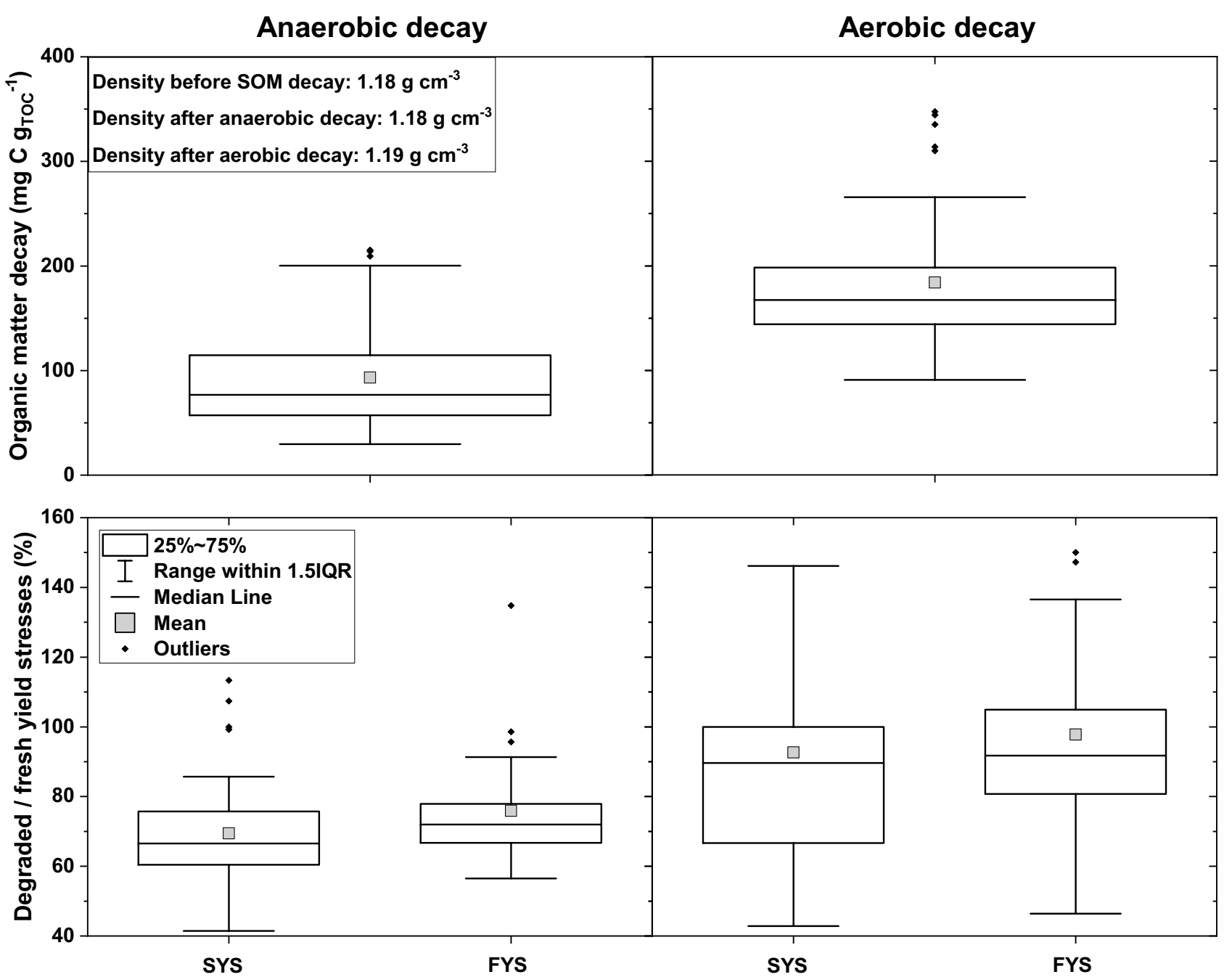

Fig. 1 Cumulative carbon release by organic matter decay over 250 days (top) and corresponding yield stress of degraded sample in relation to fresh sample (in \% of yield stress of fresh sample, Eq. (2), bottom) for anaerobic decay (AN, bottom left, $n=95$ ) and aerobic

layers fluid mud (FM), pre-consolidated sediment (PS), and consolidated sediment (CS). The largest absolute change of yield stresses $\Delta \tau_{s, f}$ (Fig. 3, top) was observed for the denser, i.e., deeper and more consolidated sediments (CS) layers. FM layers showed a larger but more scattered relative change of the static yield stresses upon decay (Fig. 3, bottom). On average, the change in fluidic yield stresses were about threefold larger than the change in static yield stresses. Considering the relative change in yield stress (Fig. 3, bottom), the change was about 60 to $90 \%$ for all layers. A few samples from FM layers showed an increase in yield stress.

Due to the fact that the absolute change in yield stresses was the largest and showed the largest range for CS layers (Fig. 3, top), data from these layers were used to investigate a possible spatial trend in more detail (Fig. 4). The change decay (AE, bottom right, $n=66$ ), all layers and locations samples in 2019 and 2020. $100 \%=$ decayed sample had the same YS value than the initial sample. $1.51 \mathrm{QR}=$ factor 1.5 of the interquartile range $(25-$ $75 \%)$

of fluidic yield stress (and also of static yield stress, not depicted) showed clear decrease from upstream to downstream locations for anaerobic SOM decay (Fig. 4, left) and a partial increase for aerobic decay (Fig. 4, right); i.e., the differences between initial yield stress and yield stress of decayed sediments were smaller at downstream locations.

The higher the extent of anaerobic SOM decay, the larger the change in fluidic yield stresses (Fig. 5). This pattern remained similar for different incubation times (21 and 250 days), during which the magnitude of change in fluidic yield stress did not change anymore. The largest spread in yield stress response to SOM decay as well as its decline appeared to result from cumulative decay of up to about $10 \mathrm{mg} \mathrm{C} \mathrm{g}_{\mathrm{TOC}^{-1}}{ }^{-1}\left(21\right.$ days) and $60 \mathrm{mg} \mathrm{C} \mathrm{g}_{\mathrm{TOC}^{-1}}{ }^{-1} 250$ days $)$ and remained on a low level beyond these thresholds. The yield stress decreased by 4 to $43 \%$ of the initial value (all 


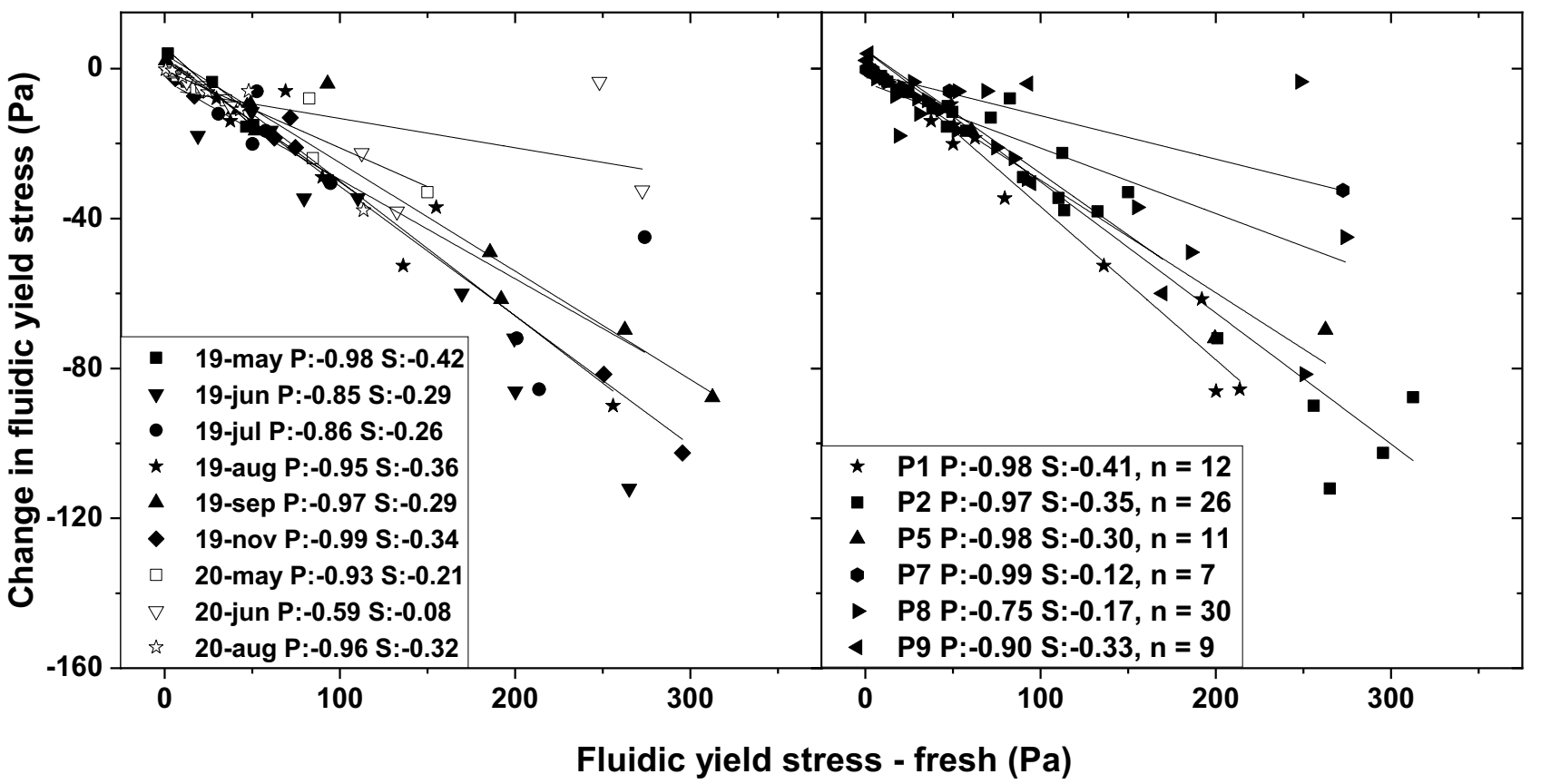

Fig. 2 Relationship between fluidic yield stress of fresh samples $\tau_{f \text { fresh }}$ and the change in yield stresses (Eq. 1) of anaerobically incubated samples (250 days) from 2019 and 2020. Left: closed sym-

incubation times) with a cumulative SOM decay between 3 and $29 \mathrm{mg} \mathrm{Cg}_{\mathrm{TOC}^{-1}}{ }^{-1}\left(21\right.$ days) and 30 and $121 \mathrm{mg} \mathrm{C}_{\mathrm{TOC}^{-1}}$ (250 days). For FM and PS layers, these trends were less pronounced; for aerobically decayed samples, these trends were not seen.

\section{Discussion}

\subsection{General effects of SOM decay on static and fluidic yield stresses}

Exhaustive decay of the degradable share of sediment organic matter led to a significant reduction in yield stresses, evidenced by a strong decrease in static and fluidic yield stresses. The effect was more pronounced for decay under anaerobic than under aerobic experimental conditions. In situ, mainly anaerobic conditions prevailed, caused by high oxygen consumption rates in the top few $\mathrm{mm}$ of the sediment deposit (Spieckermann 2021) and evidenced by negative redox potentials (Zander et al. 2020 and Table S1, supporting information). Hence, SOM degradation in the bulk sediment occurs under anaerobic conditions, leading to the release of carbon as $\mathrm{CH}_{4}$ and $\mathrm{CO}_{2}$. Both anaerobic and aerobic SOM decay clearly led to a significant decrease in yield stresses (Fig. 1); however, under aerobic conditions, the effect was less pronounced and more scattered. It is hypothesized that the decrease in yield stresses upon bols $=2019$, open symbols $=2020$, Pearson's coefficient $r(P)$, and slope (S) given per sampling date (all locations, $n=10$, left) and per location (all months, right) for chosen locations, all layers

SOM decay has a biological and a physical component, with microbial breakdown of organic bridging between particles reducing particle-particle interactions and presence of gas resulting from anaerobic decay causing the physical effect of decreasing bulk density, leading to further decline in yield stress. The later was supported by Jommi et al. (2019) who found that the presence of gas must be accounted for when studying the reduction in shear stresses by organic matter decay. It was found that the SOM decay caused a significant decrease in the rheological properties including yield stress, crossover amplitude, complex modulus, and thixotropic hysteresis area (Shakeel et al. 2022). For the given data set, the changes in density during the period of incubation observed in this study were only minor, which means that the detected changes in the rheological behavior are not caused by changes in the material's density.

In literature, higher yield stress values of fine-grained sediments were typically associated to either higher density or higher organic matter content (Van Kessel and Blom 1998; Soltanpour and Samsami 2011; Xu and Huhe 2016; Shakeel et al. 2019, 2020a, 2022) with organic matter assumed to result in a stronger structure, i.e., higher yield stresses, of fine-grained sediment (Shakeel et al. 2019). Shear stresses of long-term incubated (250 days) samples correlated best with long-term SOM degradability, seen in Fig. 5 (right), where anaerobic SOM decay led to larger change in fluidic yield stresses. During the initial decay of 10 or $60 \mathrm{mg} \mathrm{C}_{\mathrm{TOC}^{-1}}{ }^{-1}$ (corresponding to 21 or 250 days of 


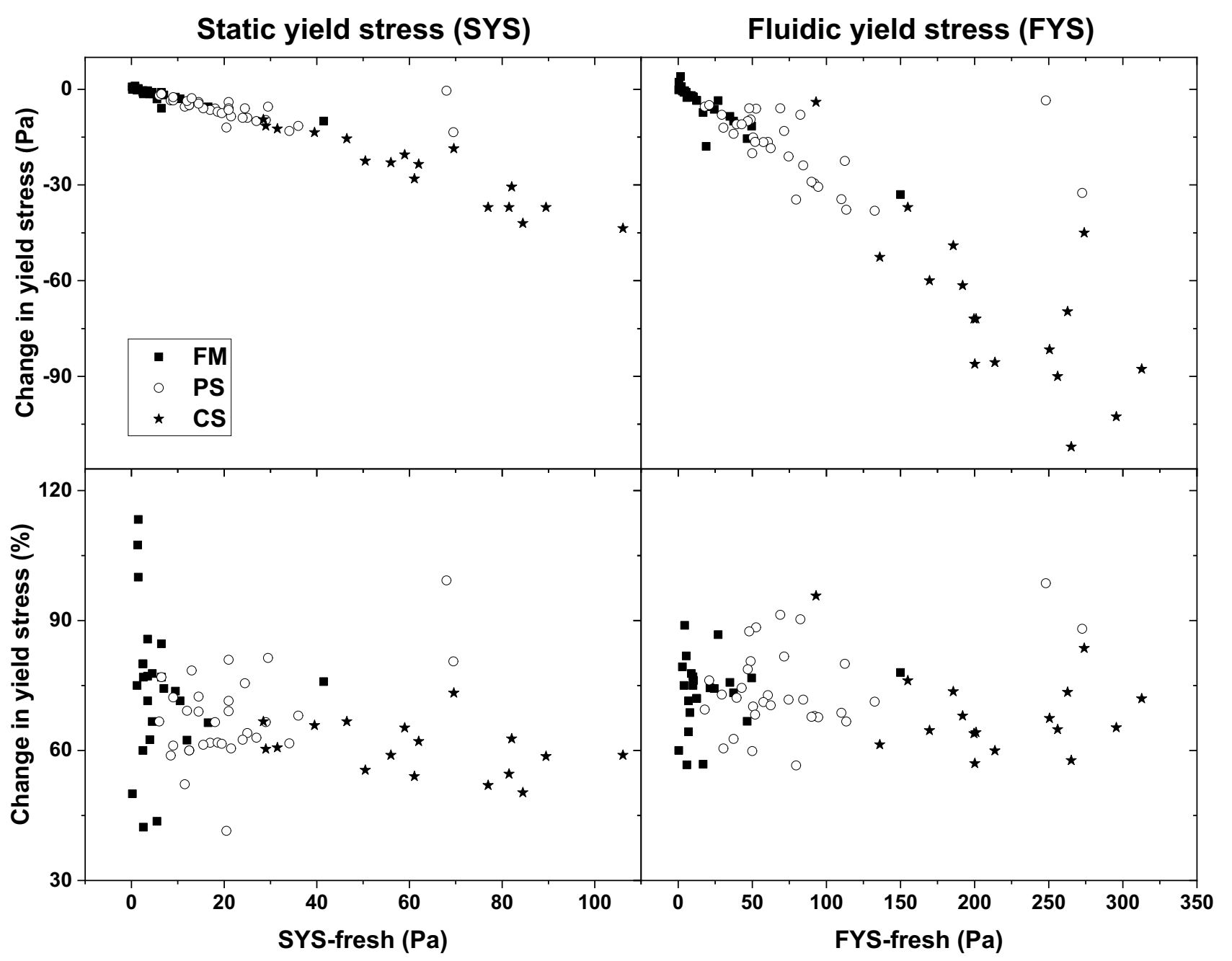

Fig. 3 Relationship between initial yield stresses of fresh samples and the change of the yield stresses upon organic matter degradation for 250 days for FM, PS, and CS layers, given in Pa (degraded-fresh,

incubation), the yield stresses decreased with an increasing amount of SOM degraded. The variability is assumed to be due to the variable amount of (easily) degradable SOM in the sample. When SOM decay progressed beyond this threshold, the yield stress did no longer decrease but remained on a low level. It is therefore concluded that the initial SOM decay is most relevant for the yield stress change, breaking up the organic bridging between particles (for a conceptual model see Shakeel et al. 2020b). In a parallel study investigating the structural recovery of sheared samples, it could be shown that after breakdown of SOM the samples exhibit a better recovery after shearing (Shakeel et al. 2022). This is due to the fact if the SOM bridges are broken, the elastic properties of the samples are greatly reduced and, in line with what is observed for sediment samples without organic matter, these samples can fully recover their pre-shearing state. This corroborates the findings of this study that the
Eq. 1, top) and in \% of fresh sample (Eq. 3, bottom). Left, static yield stress (SYS); right, fluidic yield stress (FYS), samples from all locations and all layers

decline in yield stresses is an effect of the initial phase of SOM breakdown, weakening or destroying organic bridging between mineral particles.

While the top fluidic sediment layers (FM) show the lowest absolute yield stress change, their average relative change upon SOM degradation was slightly larger than for the other layers but also scattered the most (Fig. 3). Reasons for the large scatter of the more liquid (FM) layers could be the variable degree of aging (in situ SOM decay) of FM layers and therefore changing properties during the year, or dredging interventions such as water injection dredging (WID) that lead to the formation of WID-induced FM with a different behavior than undisturbed, naturally formed FM layers. Moreover, FM layers are highly affected by the water movement (tide, large vessels, etc.). These FM layers also contain the highest share of organic carbon in the light density fraction, SOM degradability and SOM pools (Zander et al. 

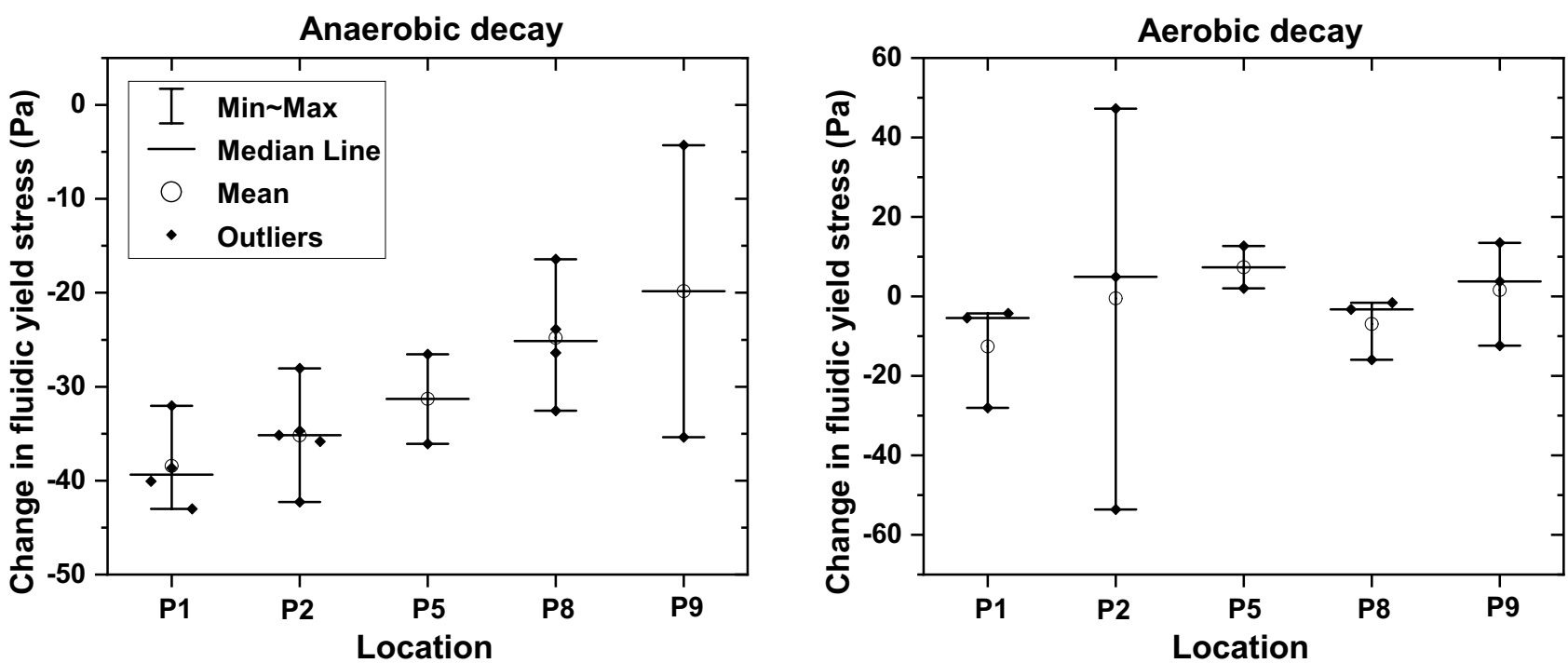

Fig. 4 Change in fluidic yield stresses $\Delta \tau_{f}$ (Eq. 1) after SOM decay (250 days) for consolidated (CS) layers from 2019 under anaerobic conditions (left) and under aerobic conditions (right) for location P1, P2, P5, P8, and P9

2022a), corroborating the relevance of easily degradable organic matter for the effects of SOM decay on the rheological behavior of sediments. This is also supported by the absolute decrease in yield stresses as a response to SOM degradation from upstream (P1) to downstream (P9) locations (shown for consolidated layers in Fig. 5), coinciding with decreasing patterns of SOM degradability, microbial biomass, and an increase of carbon bound in the hardly degradable heavy density fraction (Zander et al. 2022b). The more-dense layers (i.e., CS layers) showed the largest change between the initial and the decayed yield stress (Fig. 3). This appears plausible as more consolidated sediments have more mass, and hence more degradable carbon per unit volume than less consolidated or fluidic sediments, leading to a higher absolute reduction of yield stresses. Jommi et al.
(2019) described that gas in peat layers led to a dramatic reduction of the mobilized (load below maximum) shear strength, although the ultimate (maximum) shear strength was hardly affected.

Although the average mass removed by anaerobic SOM decay after 250 days was only $0.6 \%$ DM (15\% SOM decay with $4 \%$ TOC per DM, Zander et al. 2022a), a strong decrease in yield stresses was observed. Interestingly, under aerobic conditions $(0.8 \% \mathrm{DM})$, the influence of the SOM decay on rheological properties was less, despite the absolute mass of SOM removed being higher $(0.8 \%)$, supporting the assumption that the effect of gas bubbles formed under anaerobic conditions being responsible for an added physical effect, causing a further decline in yield stresses. Under aerobic conditions, precipitation of iron and
Fig. 5 Relationship between cumulative anaerobic SOM decay of CS layers from 2019 after 21 and 250 days and the relative change in fluidic yield stresses (degraded-fresh, in \% of the yield stress of the fresh sample, Eq. 3) upon SOM decay (250 days)

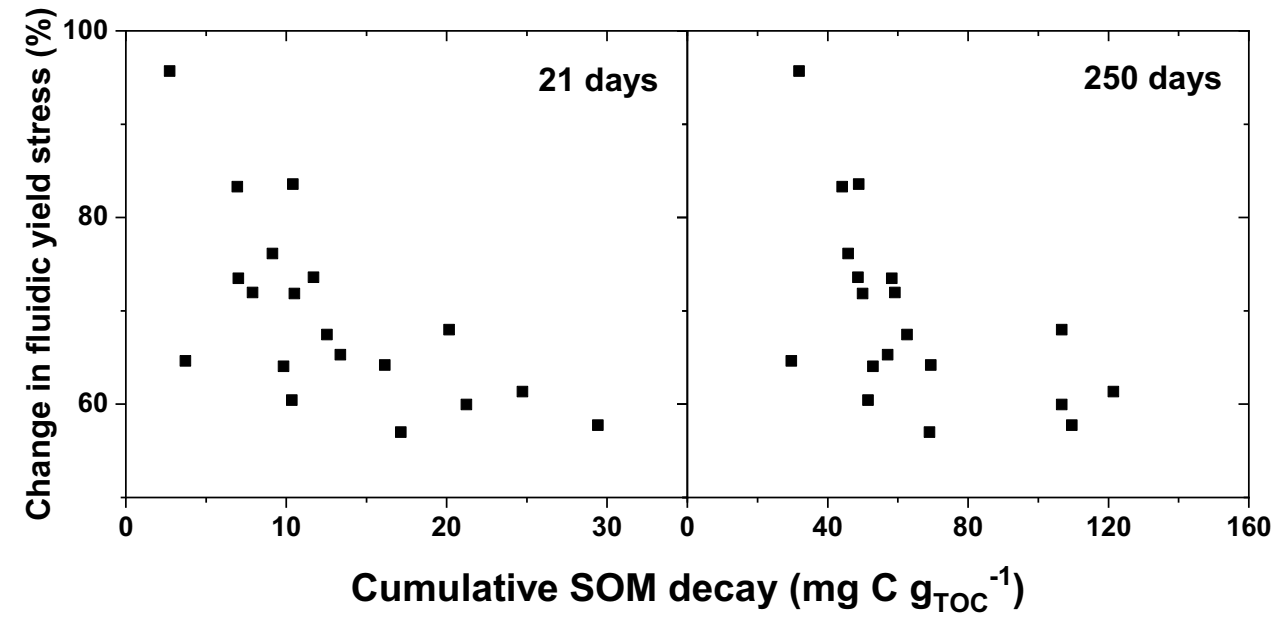


manganese oxides support cementation processes and therefore the physical stabilization of particles. This was assumed to lead to larger shear stresses of the aerobically incubated sediments.

\subsection{Seasonal and spatial trends}

The ratio between the initial yield stresses and the change in yield stresses of anaerobically incubated samples showed a linear trend for each sampling date (see Fig. 2, left), assuming that yield stresses were influenced by the seasonally changing boundary conditions affecting content and quality of organic matter, such as input of fresh SOM, SOM degradability, sedimentation rates, or dredging. Schoel et al. (2014) found seasonally changing (easily degradable) algae biomass concentrations in the Elbe River, and it were observed seasonal changes in easily degradable SOM pools (Zander et al. 2022b). The more negative slopes of changes in yield stress upon SOM decay over yield stress of the freshly sampled sediments in 2019 compared to 2020 (Fig. 2, left) suggest that in 2019, the sediments in the investigation area contained a larger share of easily degradable organic matter. Although locations P2 and P8 showed similar TOC and clay content (Table S1), they showed different behavior in yield stresses due to their different amount of easily degradable organic matter available at these locations. At upstream location P2, a larger share of upstream driven SOM reached this location compared to the more downstream-input driven location P8 (also explained in Zander et al. 2020). At upstream locations, a higher concentration of algae in the water column, represented by the chlorophyll a concentration (Zander et al. 2020), was detected, the measured degradability of SOM was higher, and a larger share of the light density fraction was found. Therefore, it is assumed that a larger share of easily degradable SOM, i.e., low molecular weight substances like cellulose, and a smaller share of slowly degradable SOM, i.e., high molecular substances like lignin, are present at upstream locations.

The spatial trends of the yield stress change (Fig. 4) coincide with the known stratification of organic matter degradability, with higher degradability in upstream locations and lower degradability in downstream samples. For all locations, the slopes for the ratio between the initial yield stresses and the change in yield stresses of anaerobically incubated samples were negative (Fig. 2, right), meaning that the SOM decay led to a decrease in yield stresses. Upstream locations (P1 and P2) had the most negative slopes and showed the greatest absolute changes in yield stress for consolidated layers (Fig. 4, left), reflecting the higher share of easily available organic matter and the largest amount of degradable carbon at these sites (Zander et al. 2022b). Per location, the slopes of the lines, correlating the change in fluidic yield stress (degradedinitial) and the initial fluidic yield stress, varied within the range of -0.12 and -0.41 (Fig. 2, right). This shows that the influence of organic matter degradation on sediment strength differs per sampling location, too. At the upstream locations (P1 and P2), more easily degradable SOM was available (Zander et al. 2020), and therefore, the differences of the fluidic yield stress between fresh and degraded samples were larger (Fig. 4).

\section{Conclusions and outlook}

This study showed that under anaerobic conditions, shear strengths were decreased strongly after microbial SOM decay, whereas the effect under aerobic conditions was lower, in spite of the fact that the absolute amount of organic matter decayed under aerobic conditions was higher. It is concluded that this effect reflects the combination of the biological effect of SOM degradation and the physical effect of entrapment of gas bubbles in the sediment, furthering the reduction of strength. Degradation of organic matter significantly affects sediment strength, especially under the anaerobic conditions prevailing in situ, even when the mass of organic matter removed is only little, here around $0.6 \%$ of dry matter. The effect sets in predominantly in the initial phases of degradation, which are assumed to be responsible for the break-down of organic bridging between mineral particles, thereby reducing the particle-particle interactions. A larger absolute change in yield stresses was seen with increasing depth, with gassy consolidated (CS) layers showing the largest decrease in yield stresses, owing to the fact that more consolidated sediments contain more mass, and hence degradable carbon per unit volume. The CS layers showed the most distinct spatial trend, i.e., the decreasing change in static and fluidic yield stresses from upstream to downstream, coinciding with a gradient of decreasing SOM degradability. Hence, larger changes in yield stresses were seen at locations with high SOM decay, highlighting sitespecific response of the rheological behavior due to sitespecific availability of degradable organic matter. Seasonal trends with all sites along the transect following similar trends were also found, indicating that the investigation area as a whole is impacted by temporally changing factors such as the availability of easily degradable organic matter.

Supplementary information The online version contains supplementary material available at https://doi.org/10.1007/s11368-022-03155-6.

Acknowledgements Deltares is acknowledged for the use of the HAAKE MARS I rheometer. The authors thank Dr. Susan Buisma-Yi for proofreading the manuscript. 
Funding This study was funded by Hamburg Port Authority and carried out within the project BIOMUD and RHEOMUD, members of the MUDNET academic network www.tudelft.nl/mudnet/.

\section{Declarations}

Conflict of interest The authors declare no competing interests.

Open Access This article is licensed under a Creative Commons Attribution 4.0 International License, which permits use, sharing, adaptation, distribution and reproduction in any medium or format, as long as you give appropriate credit to the original author(s) and the source, provide a link to the Creative Commons licence, and indicate if changes were made. The images or other third party material in this article are included in the article's Creative Commons licence, unless indicated otherwise in a credit line to the material. If material is not included in the article's Creative Commons licence and your intended use is not permitted by statutory regulation or exceeds the permitted use, you will need to obtain permission directly from the copyright holder. To view a copy of this licence, visit http://creativecommons.org/licenses/by/4.0/.

\section{References}

Baldock JA, Skjemstad JO (2000) Role of the soil matrix and minerals in protecting natural organic materials against biological attack. Organic Geochem 31:697-710. https://doi.org/10.1016/S01466380(00)00049-8

Deng Z, He Q, Safar Z, Chassagne C (2019) The role of algae in fine sediment flocculation: In-situ and laboratory measurements. Mar Geol 413:71-84. https://doi.org/10.1016/j.margeo.2019.02.003

Gao J, Mikutta R, Jansen B, Guggenberger G, Vogel C, Kalbitz K (2019) The multilayer model of soil mineral-organic interfaces - a review. J Plant Nutr Soil Sci 000:1-15. https://doi.org/10.1002/jpln.201900530

Jommi C, Muraro S, Trivellato E, Zwanenburg C (2019) Experimental results on the influence of gas on the mechanical response of peats. Géotechnique 69(9):753-766. https://doi.org/10.1680/jgeot.17.P.148

Lagaly G, Dékány I (2013) Chapter 8 - colloid clay science. In: Bergaya F, Lagaly G (eds) Developments in Clay Science. Elsevier, 243-345

Schoel A, Hein B, Wyrwa J, Kirchesch V (2014) Modelling water quality in the Elbe and its estuary -large scale and long-term applications with focus on the oxygen budget of the estuary. Die Kueste 81:203-232. https://hdl.handle.net/20.500.11970/101692. Accessed 6 Dec 2021

Shakeel A, Kirichek A, Chassagne C (2019) Is density enough to predict the rheology of natural sediments? Geo-Mar Lett 39:427-434. https://doi.org/10.1007/s00367-019-00601-2
Shakeel A, Kirichek A, Chassagne C (2020a) Rheological analysis of mud from Port of Hamburg, Germany. J Soils Sediments 20:25532562. https://doi.org/10.1007/s11368-019-02448-7

Shakeel A, Kirichek A, Chassagne C (2020b) Yield stress measurements of mud sediments using different rheological methods and geometries: an evidence of two-step yielding. Marine Geol 427:106247. https://doi.org/10.1016/j.margeo.2020.106247

Shakeel A, Zander F, de Kerk J-W, Kirichek A, Gebert J, Chassagne C (2022) Effect of organic matter degradation in cohesive sediment: a detailed rheological analysis. J Soils Sediments. https:// doi.org/10.1007/s11368-022-03156-5. (accepted for publication 30 January 2022)

Six J, Paustian K (2014) Aggregate-associated soil organic matter as an ecosystem property and a measurement tool. Soil Biol Biochem 68:A4-A9. https://doi.org/10.1016/j.soilbio.2013.06.014

Soltanpour M, Samsami F (2011) A comparative study on the rheology and wave dissipation of kaolinite and natural Hendijan Coast mud, the Persian Gulf. Ocean Dyn 61:295-309. https://doi.org/10.1007/ s10236-011-0378-7

Spieckermann MJ (2021) Controls of oxygen consumption of sediments in the Upper Elbe Estuary. Dissertation. University of Hamburg. Hamburger Bodenkundliche Arbeiten 101. https://ediss.sub. uni-hamburg.de/handle/ediss/9129. Accessed 5 Dec 2021

Van Kessel T, Blom C (1998) Rheology of cohesive sediments: comparison between a natural and an artificial mud. J Hydraul Res 36:591-612. https://doi.org/10.1080/00221689809498611

Vannote RL, Minshall WG, Cummins KW, Sedell JR, Cushing CE (1980) The river continuum concept. Can J Fish Aquat Sci 37:130-137. https://doi.org/10.1139/f80-017

Wurpts R, Torn P (2005) 15 years experience with fluid mud: definition of the nautical bottom with rheological parameters. Terra Et Aqua 99:22-32

Xu J, Huhe A (2016) Rheological study of mudflows at Lianyungang in China. Int J Sediment Res 31:71-78. https://doi.org/10.1016/j. ijsrc.2014.06.002

Zander F, Heimovaara T, Gebert J (2020) Spatial variability of organic matter degradability in tidal Elbe sediments. J Soils Sediments 20:2573-2587. https://doi.org/10.1007/s11368-020-02569-4

Zander F, Gröngröft A, Eschenbach A, Heimovaara T, Gebert J (2022a) Organic matter pools in sediments of the tidal Elbe river. Limnol (submitted)

Zander F, Comans RNJ, Gebert J (2022b) Linking patterns of density, thermometric and carbon stable isotope fractions of organic matter to its lability in sediments of the tidal Elbe river. Appl Geochem (submitted)

Publisher's Note Springer Nature remains neutral with regard to jurisdictional claims in published maps and institutional affiliations.

\section{Authors and Affiliations}

\section{F. Zander ${ }^{1}$ (D) A. Shakeel ${ }^{2,3} \cdot$ A. Kirichek ${ }^{2} \cdot$ C. Chassagne ${ }^{2} \cdot$ J. Gebert $^{1}$}
A. Shakeel
A.Shakeel@tudelft.nl
A. Kirichek
O.Kirichek@tudelft.nl
C. Chassagne
C.Chassagne@tudelft.nl

J. Gebert

J.Gebert@tudelft.nl

1 Faculty of Civil Engineering and Geosciences, Department of Geoscience and Engineering, Section of Geo-Engineering, Delft University of Technology, Stevinweg 1, 2628 CN Delft, Netherlands 
2 Faculty of Civil Engineering and Geosciences, Department of Hydraulic Engineering, Section of Environmental Fluid Mechanics, Delft University of Technology, Stevinweg 1, 2628 CN Delft, Netherlands
3 Department of Chemical, Polymer \& Composite Materials Engineering, University of Engineering \& Technology, KSK Campus, Lahore 54890, Pakistan 